\title{
Imaging of a Dissipative Layer in a Random Medium using a Time Reversal Method
}

\author{
Jean-Pierre Fouque ${ }^{1}$, Josselin Garnier ${ }^{2}$, André Nachbin ${ }^{3}$, and Knut Sølna ${ }^{4}$ \\ 1 Department of Mathematics, North Carolina State University, Raleigh NC \\ 27695-8205. fouque@math.ncsu.edu \\ 2 Instituto de Matemática Pura e Aplicada, Est. D. Castorina 110, Jardim \\ Botânico, Rio de Janeiro, RJ 22460-320, Brazil. nachbin@impa.br \\ 3 Laboratoire de Statistique et Probabilités, Université Paul Sabatier, 118 Route \\ de Narbonne, 31062 Toulouse Cedex 4, France. garnier@cict.fr \\ 4 Department of Mathematics, University of California, Irvine CA 92697. \\ ksolna@math.uci.edu
}

Summary. In this paper time reversal of acoustic waves in a dissipative random one-dimensional medium is analyzed. It is shown that time reversal can be used as an efficient and statistically stable method to image a dissipative layer embedded in a random scattering medium. The quantities needed to achieve this goal appear as the solutions of a system of transport equations which are solved by a Monte Carlo method.

\section{Introduction}

Time-reversal refocusing for waves propagating in inhomogeneous media have been recently observed and studied experimentally in various contexts, e.g. ultrasound, underwater acoustics, see for instance the review [Fin99]. Important potential applications have been proposed in various fields, for instance imaging [PKC02, FMT03] and communication [DTR03]. A time-reversal mirror is, roughly speaking, a device which is capable of receiving a signal in time, keeping it in memory and sending it back into the medium in the reversed direction of time. The main effect is the refocusing of the scattered signal after time-reversal in a random medium. Surprisingly, the refocused pulse shape only depends on the statistical properties of the random medium, and not on the particular realization of the medium. The full mathematical understanding, meaning both modeling of the physical problem and derivation of the time-reversal effect, is a complex problem. The study of the one-dimensional linear case is now well understood [CF97, Sol03, FS03] as well as the three-dimensional waves in the parabolic or paraxial regime [BPR02, BPZ02, PRS03]. In this paper we generalize the model considered so far by introducing absorption modeled by a linear dissipative term in the 
acoustic equations. We show that time-reversal is still efficient in refocusing waves despite the loss of energy due to absorption.

The paper is organized as follows. In Sections 2-4 we derive integral representations of the refocused pulse shapes in terms of reflection coefficients for the wave modes. Section 5 is devoted to the thorough analysis of timereversal experiments in reflection in random and dissipative media. In Section 6 we use a probabilistic interpretation of the limit solution to analyze different configurations by Monte Carlo (MC) simulations.

\section{The Acoustic Model}

We consider the acoustic wave equation

$$
\begin{aligned}
& \frac{1}{K} \frac{\partial p}{\partial t}+\frac{\partial u}{\partial z}=0, \\
& \rho \frac{\partial u}{\partial t}+\frac{\partial p}{\partial z}+\sigma u=0
\end{aligned}
$$

where $p$ is the pressure and $u$ is the velocity, $z$ and $t$ are the space and time coordinates, respectively, $\sigma$ is the dissipation of the medium, $\rho$ is the density, and $K$ is the bulk modulus. The fluctuations of the medium parameters are described by

$$
\begin{gathered}
\rho= \begin{cases}1+\nu\left(\frac{z}{\varepsilon^{2}}\right) & \text { if }-L<z<0, \\
1 & \text { if } z>0 \text { or } z<-L,\end{cases} \\
\frac{1}{K}= \begin{cases}1+\eta\left(\frac{z}{\varepsilon^{2}}\right) & \text { if }-L<z<0, \\
1 & \text { if } z>0 \text { or } z<-L,\end{cases} \\
\sigma= \begin{cases}\sigma\left(z, \frac{z}{\varepsilon^{2}}\right) & \text { if }-L<z<0, \\
0 & \text { if } z>0 \text { or } z<-L .\end{cases}
\end{gathered}
$$

The dimensionless small parameter $\varepsilon^{2}$ characterizes the ratio of the correlation radius of the fluctuations of the medium and the typical size of the medium. The fluctuations of the density and the bulk modulus are modeled by the zero-mean stationary random process $(\eta, \nu)(z)$. The processes $\eta$ and $\nu$ are assumed to be bounded by a deterministic constant less than 1 and to have strong ergodic properties. We may think for instance that $(\eta, \nu)$ is a function of a Markov, stationary, ergodic process on a compact space satisfying the Fredholm alternative [Pap78]. We can also work with the class of the $\phi$-mixing processes with $\phi \in L^{1 / 2}$ [Kus84]. The dissipation coefficient is a nonnegative function. It may vary rapidly at scale $\varepsilon^{2}$, but it may also have slow variations at scale one. More exactly, we assume that, for any $z \in[-L, 0], \zeta \mapsto \sigma(z, \zeta)$ is a nonnegative stationary mixing process with mean $\bar{\sigma}(z)=\mathbb{E}[\sigma(z, \zeta)]$. We think in particular at the two following configurations. 
1. The dissipation coefficient has a stationary statistical distribution (this will be our reference framework):

$$
\sigma\left(z, \frac{z}{\varepsilon^{2}}\right)=\sigma_{0}\left(\frac{z}{\varepsilon^{2}}\right)
$$

where $\sigma_{0}$ is a nonnegative stationary ergodic random process with mean $\bar{\sigma}_{0}=\mathbb{E}\left[\sigma_{0}(\zeta)\right]$.

2. The dissipation coefficient has a different behavior inside some embedded layer $\left[z_{0}, z_{1}\right],-L<z_{0}<z_{1}<0$,

$$
\sigma\left(z, \frac{z}{\varepsilon^{2}}\right)= \begin{cases}\sigma_{1}\left(\frac{z}{\varepsilon^{2}}\right) & \text { if } z_{0}<z<z_{1} \\ \sigma_{0}\left(\frac{z}{\varepsilon^{2}}\right) & \text { if } z_{1} \leq z \leq 0 \text { or }-L \leq z \leq z_{0}\end{cases}
$$

where $\sigma_{0}$ and $\sigma_{1}$ are two nonnegative stationary ergodic random processes. We denote by $\bar{\sigma}_{j}$ their respective means

$$
\bar{\sigma}_{j}=\mathbb{E}\left[\sigma_{j}(\zeta)\right]
$$

and we assume that $\bar{\sigma}_{1} \neq \bar{\sigma}_{0}$. One goal of the paper is to detect the layer $\left[z_{0}, z_{1}\right]$.

We consider the problem on the finite slab $-L \leq z \leq 0$ where boundary conditions are imposed at $-L$ and 0 corresponding to a left-going pulse entering the slab from the right at $z=0$. We consider an incoming pulse whose typical wavelength is of order $\varepsilon$, that is to say small compared to the size of the medium, but large with respect to the correlation length of the random medium:

$$
f^{\varepsilon}(t)=f\left(\frac{t}{\varepsilon}\right)
$$

\section{Propagator Formulation}

In this section we first express the scattering problem as a two point boundary value problem in the frequency domain, and then rewrite it as an initial value problem in terms of the propagator. This study follows the lines of the analysis carried out in Ref. [AKP91].

\subsection{Mode Propagation in the Frequency Domain}

We consider the random acoustic equation (1-2) and take the scaled time Fourier transform

$$
\hat{u}^{\varepsilon}(\omega, z)=\frac{1}{2 \pi \varepsilon} \int u(t, z) e^{-\frac{i \omega t}{\varepsilon}} d t, \quad \hat{p}^{\varepsilon}(\omega, z)=\frac{1}{2 \pi \varepsilon} \int p(t, z) e^{-\frac{i \omega t}{\varepsilon}} d t,
$$

so that the system reduces to a set of ordinary differential equations: 


$$
\begin{aligned}
& \frac{\partial \hat{u}^{\varepsilon}}{\partial z}+\frac{i \omega}{\varepsilon}\left(1+\eta\left(\frac{z}{\varepsilon^{2}}\right)\right) \hat{p}^{\varepsilon}=0, \\
& \frac{\partial \hat{p}^{\varepsilon}}{\partial z}+\frac{i \omega}{\varepsilon}\left(1+\nu\left(\frac{z}{\varepsilon^{2}}\right)\right) \hat{u}^{\varepsilon}+\sigma \hat{u}^{\varepsilon}=0 .
\end{aligned}
$$

We decompose the wave into right-going modes $a^{\varepsilon}$ and left-going modes $b^{\varepsilon}$

$$
\begin{aligned}
& a^{\varepsilon}(\omega, z)=\frac{1}{2}\left(\hat{p}^{\varepsilon}(\omega, z)+\hat{u}^{\varepsilon}(\omega, z)\right) e^{\frac{i \omega z}{\varepsilon}}, \\
& b^{\varepsilon}(\omega, z)=\frac{1}{2}\left(\hat{p}^{\varepsilon}(\omega, z)-\hat{u}^{\varepsilon}(\omega, z)\right) e^{-\frac{i \omega z}{\varepsilon}},
\end{aligned}
$$

which satisfy the linear equation

$$
\frac{\partial}{\partial z}\left(\begin{array}{l}
a^{\varepsilon} \\
b^{\varepsilon}
\end{array}\right)(\omega, z)=Q^{\varepsilon}(\omega, z)\left(\begin{array}{l}
a^{\varepsilon} \\
b^{\varepsilon}
\end{array}\right)(\omega, z)
$$

where the complex $2 \times 2$ matrix $Q^{\varepsilon}$ is given by:

$$
Q^{\varepsilon}(\omega, z)=\frac{i \omega}{2}\left(\begin{array}{cc}
-m^{\varepsilon}(z) & -n^{\varepsilon}(z) e^{\frac{2 i \omega z}{\varepsilon}} \\
n^{\varepsilon}(z) e^{-\frac{2 i \omega z}{\varepsilon}} & m^{\varepsilon}(z)
\end{array}\right)+\frac{\sigma^{\varepsilon}(z)}{2}\left(\begin{array}{cc}
-1 & e^{\frac{2 i \omega z}{\varepsilon}} \\
-e^{-\frac{2 i \omega z}{\varepsilon}} & 1
\end{array}\right)
$$

with

$$
\begin{aligned}
& m(z)=\eta(z)+\nu(z), \quad n(z)=\eta(z)-\nu(z), \\
& m^{\varepsilon}(z)=\frac{1}{\varepsilon} m\left(\frac{z}{\varepsilon^{2}}\right), \quad n^{\varepsilon}(z)=\frac{1}{\varepsilon} n\left(\frac{z}{\varepsilon^{2}}\right), \quad \sigma^{\varepsilon}(z)=\sigma\left(z, \frac{z}{\varepsilon^{2}}\right) .
\end{aligned}
$$

\subsection{Boundary Values}

We assume that a left-going pulse is incoming from the right and is scattered into a reflected wave at $z=0$ and a transmitted wave at $z=-L$ (see Figure 1 ). The incoming pulse shape is given by the pressure function $f(t / \varepsilon)$ where

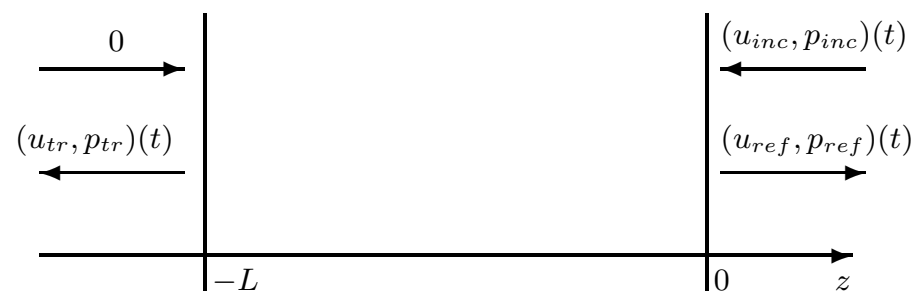

Fig. 1. Scattering problem.

$f$ is assumed to be a $L^{1}$ function compactly supported in the Fourier domain:

$$
u_{i n c}(t, z=0)=-\int \hat{f}(\omega) e^{\frac{i \omega t}{\varepsilon}} d \omega, \quad p_{\text {inc }}(t, z=0)=\int \hat{f}(\omega) e^{\frac{i \omega t}{\varepsilon}} d \omega,
$$


where $\hat{f}$ is the standard Fourier transform

$$
\hat{f}(\omega)=\frac{1}{2 \pi} \int f(t) e^{-i \omega t} d t .
$$

We also impose a radiation condition at $-L$ corresponding to the absence of right-going wave at the left hand-side of the slab $[-L, 0]$. The two-point boundary value problem consisting of the system (8) for $z \in[-L, 0]$ together with the conditions:

$$
b^{\varepsilon}(\omega, z=0)=\hat{f}(\omega), \quad a^{\varepsilon}(\omega, z=-L)=0
$$

is then well-posed.

\subsection{Propagator}

It is convenient to transform the two-point boundary value problem into an initial value problem by introducing the propagator $Y^{\varepsilon}(\omega,-L, z)$ which is a complex $2 \times 2$ matrix solution of

$$
\frac{\partial Y^{\varepsilon}}{\partial z}(\omega,-L, z)=Q^{\varepsilon}(\omega, z) Y^{\varepsilon}(\omega,-L, z), \quad Y^{\varepsilon}(\omega,-L, z=-L)=I d_{\mathbb{C}^{2}},
$$

such that

$$
Y^{\varepsilon}(\omega,-L, z)\left(\begin{array}{c}
a^{\varepsilon}(\omega,-L) \\
b^{\varepsilon}(\omega,-L)
\end{array}\right)=\left(\begin{array}{l}
a^{\varepsilon}(\omega, z) \\
b^{\varepsilon}(\omega, z)
\end{array}\right) .
$$

The propagator matrix $Y^{\varepsilon}$ has the form

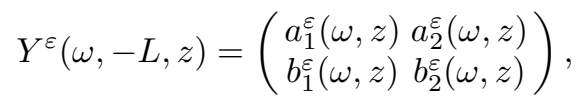

where the column vector $\left(a_{1}^{\varepsilon}, b_{1}^{\varepsilon}\right)^{T}$, resp. $\left(a_{2}^{\varepsilon}, b_{2}^{\varepsilon}\right)^{T}$, is solution of equation (8) with the initial conditions:

$$
\begin{array}{ll}
a_{1}^{\varepsilon}(\omega, z=-L)=1, & b_{1}^{\varepsilon}(\omega, z=-L)=0, \\
a_{2}^{\varepsilon}(\omega, z=-L)=0, & b_{2}^{\varepsilon}(\omega, z=-L)=1,
\end{array}
$$

respectively. We can now define the transmission and reflection coefficients $T^{\varepsilon}(\omega,-L, z)$ and $R^{\varepsilon}(\omega,-L, z)$, respectively, for a slab $[-L, z]$ by (see also Figure 2):

$$
Y^{\varepsilon}(\omega,-L, z)\left(\begin{array}{c}
0 \\
T^{\varepsilon}(\omega,-L, z)
\end{array}\right)=\left(\begin{array}{c}
R^{\varepsilon}(\omega,-L, z) \\
1
\end{array}\right) .
$$

In terms of the propagator entries they are given given by:

$$
R^{\varepsilon}(\omega,-L, z)=\frac{a_{2}^{\varepsilon}}{b_{2}^{\varepsilon}}(\omega, z), \quad T^{\varepsilon}(\omega,-L, z)=\frac{1}{b_{2}^{\varepsilon}}(\omega, z),
$$




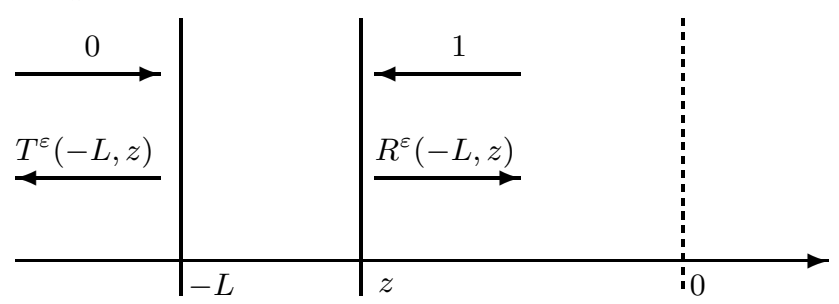

Fig. 2. Reflection and transmission coefficients.

and the reflection coefficient satisfies the closed form nonlinear Riccati differential equation:

$$
\begin{aligned}
\frac{\partial R^{\varepsilon}}{\partial z}= & -i \omega m^{\varepsilon}(z) R^{\varepsilon}-\frac{i \omega}{2} e^{-\frac{2 i \omega z}{\varepsilon}} n^{\varepsilon}(z)\left(R^{\varepsilon}\right)^{2}-\frac{i \omega}{2} e^{\frac{2 i \omega z}{\varepsilon}} n^{\varepsilon}(z) \\
& -\sigma^{\varepsilon}(z) R^{\varepsilon}+\frac{\sigma^{\varepsilon}(z)}{2}\left(e^{\frac{2 i \omega z}{\varepsilon}}+e^{-\frac{2 i \omega z}{\varepsilon}}\left(R^{\varepsilon}\right)^{2}\right)
\end{aligned}
$$

with the initial condition at $z=-L: R^{\varepsilon}(\omega,-L, z=-L)=0$. The reflected wave at time $t$, denoted by $\left(u_{r e f}, p_{r e f}\right)$, is a right-going wave whose pressure field admits the following integral representation in terms of the reflection coefficient:

$$
p_{\text {ref }}(t, z=0)=\int \hat{f}(\omega) R^{\varepsilon}(\omega,-L, 0) e^{\frac{i \omega t}{\varepsilon}} d \omega .
$$

Remark: We can give a rapid comment on the role of dissipation. The vectors satisfy $\partial_{z}\left(\left|a_{j}^{\varepsilon}\right|^{2}-\left|b_{j}^{\varepsilon}\right|^{2}\right)=-\sigma^{\varepsilon}(z)\left|a_{j}^{\varepsilon} e^{-i \omega z / \varepsilon}-b_{j}^{\varepsilon} e^{i \omega z / \varepsilon}\right|^{2}$ and thus $\left|a_{2}^{\varepsilon}\right|^{2}(z)+1 \leq\left|b_{2}^{\varepsilon}\right|^{2}(z)$. By Eq. (15) this implies the relation expressing the dissipation of energy

$$
\left|R^{\varepsilon}\right|^{2}+\left|T^{\varepsilon}\right|^{2} \leq 1
$$

A strict inequality means that some energy is lost during the scattering of the wave by the random dissipative slab. Eq. (18) also implies the uniform boundedness of the reflection coefficient.

\section{Time Reversal}

In the first step of the time reversal procedure, the time-reversal mirror (TRM) is used as a passive device to record the reflected signal at $z=0$ up to a certain time. It turns out that as $\varepsilon \rightarrow 0$ the interesting asymptotic regime arises when the signal is recorded up to a large time of order 1 which we denote by $t_{1}$ with $t_{1}>0$. Thus, the recorded signal is of the form

$$
p_{\text {rec }}(t)=p_{\text {ref }}(t) G_{t_{1}}(t),
$$

where the the support of the cut-off function $G_{t_{1}}$ is included in $\left[0, t_{1}\right]$ and $p_{r e f}$ is given by (17). In the second step the TRM is used as an active device: it 
time reverses the recorded signal and sends it back into the medium. Observe that the part of the wave which came in last will go out first. It also changes the sign of the recorded velocity so as to generate a pure left-going wave. Thus, the time-reversed pressure wave sent back into the medium is given by:

$$
\begin{aligned}
p_{\text {inc }(T R)}(t, z=0) & =p_{\text {rec }}\left(t_{1}-t\right) \\
& =\frac{1}{\varepsilon} \iint e^{\frac{i \omega\left(t_{1}-t\right)}{\varepsilon}} \hat{p}_{r e f}^{\varepsilon}\left(\omega^{\prime}\right) \hat{G}_{t_{1}}\left(\frac{\omega-\omega^{\prime}}{\varepsilon}\right) d \omega^{\prime} d \omega,
\end{aligned}
$$

where $T R$ stands for "Time Reversal" and $\hat{G}_{t_{1}}$ is the Fourier transform of $G_{t_{1}}$. Using the fact that $p_{i n c(T R)}$ is real valued, we can also write:

$$
p_{i n c(T R)}(t, z=0)=\frac{1}{\varepsilon} \iint e^{\frac{i \omega\left(t-t_{1}\right)}{\varepsilon}} \overline{\hat{p}}_{r e f}^{\varepsilon}\left(\omega^{\prime}\right) \overline{\hat{G}}_{t_{1}}\left(\frac{\omega-\omega^{\prime}}{\varepsilon}\right) d \omega^{\prime} d \omega .
$$

This new incoming signal re-propagates into the same medium and generates a new reflected signal which we observe at the time $t_{2}+\varepsilon t$, that is, around the time $t_{2}$ in the scale of the initial pulse. In terms of the reflection coefficients $R^{\varepsilon}$ the observed reflected signal is given by

$$
p_{r e f(T R)}\left(t_{2}+\varepsilon t, z=0\right)=\int \hat{p}_{i n c(T R)}^{\varepsilon}(\omega) R^{\varepsilon}(\omega,-L, 0) e^{\frac{i \omega t_{2}}{\varepsilon}+i \omega t} d \omega .
$$

Substituting the expression of $\hat{p}_{i n c(T R)}^{\varepsilon}$ into this equation yields the following representation of the reflected signal:

$$
\begin{aligned}
p_{r e f(T R)}\left(t_{2}+\varepsilon t, z=0\right)=\frac{1}{\varepsilon} \iint & e^{i \omega t} e^{\frac{i \omega\left(t_{2}-t_{1}\right)}{\varepsilon}} \overline{\hat{f}}\left(\omega^{\prime}\right) \overline{\hat{G}}_{t_{1}}\left(\frac{\omega-\omega^{\prime}}{\varepsilon}\right) \\
& \times R^{\varepsilon}(\omega,-L, 0) \overline{R^{\varepsilon}}\left(\omega^{\prime},-L, 0\right) d \omega^{\prime} d \omega .
\end{aligned}
$$

Motivated by the scaled argument of $\hat{G}_{t_{1}}$ we get by the change of variable $\omega^{\prime}=\omega-\varepsilon h:$

$$
\begin{aligned}
p_{\text {ref }(T R)}\left(t_{2}+\varepsilon t, z=0\right)= & \iint e^{i \omega t} e^{\frac{i \omega\left(t_{2}-t_{1}\right)}{\varepsilon}} \overline{\hat{f}}(\omega-\varepsilon h) \overline{\hat{G}}_{t_{1}}(h) \\
& \times R^{\varepsilon}(\omega,-L, 0) \overline{R^{\varepsilon}}(\omega-\varepsilon h,-L, 0) d h d \omega .
\end{aligned}
$$

The rapid phase $\exp \left(i \omega\left(t_{2}-t_{1}\right) / \varepsilon\right)$ averages out this integral except when $t_{2}=t_{1}$. This means that refocusing can be observed only at the time $t_{2}=t_{1}$. The precise description of the refocused pulse will be carried out in the next section.

\section{The Refocused Pulse}

The refocused pulse observed at $z=0$ and at time $t_{1}+\varepsilon t$ is given by 


$$
\begin{aligned}
p_{r e f(T R)}\left(t_{1}\right. & +\varepsilon t)=\iint e^{i \omega t} e^{i \varepsilon h t / 2} \overline{\hat{f}}(\omega-\varepsilon h / 2) \overline{\hat{G}}_{t_{1}}(h) \\
& \times R^{\varepsilon}(\omega+\varepsilon h / 2,-L, 0) \overline{R^{\varepsilon}}(\omega-\varepsilon h / 2,-L, 0) d h d \omega .
\end{aligned}
$$

This section is devoted to the proof of the convergence of the refocused pulse shape to a deterministic shape as $\varepsilon \rightarrow 0$.

\subsection{Tightness}

We first address the tightness of the process.

Lemma 1. The refocused pulse $\left(\left(p_{\text {ref(TR) }}^{\varepsilon}\left(t_{1}+\varepsilon t\right)\right)_{-\infty<t<\infty}\right)_{\varepsilon>0}$ is a tight (i.e. weakly compact) family in the space of continuous trajectories equipped with the sup norm.

Proof. We must show that, for any $\delta>0$, there exists a compact subset $K$ of the space of continuous bounded functions such that:

$$
\sup _{\varepsilon>0} \mathbb{P}\left(p_{r e f(T R)}^{\varepsilon}\left(t_{1}+\varepsilon \cdot\right) \in K\right) \geq 1-\delta
$$

On the one hand the dissipation relation (18) yields that $\left|R^{\varepsilon}\right| \leq 1$ and $p_{\text {ref(TR) }}^{\varepsilon}\left(t_{1}+\varepsilon t\right)$ is uniformly bounded by:

$$
\left|p_{r e f(T R)}^{\varepsilon}\left(t_{1}+\varepsilon t\right)\right| \leq \int|\hat{f}(\omega)| d \omega \times \int\left|\hat{G}_{t_{1}}(h)\right| d h
$$

On the other hand the modulus of continuity

$$
M^{\varepsilon}(\delta)=\sup _{\left|s_{1}-s_{2}\right| \leq \delta}\left|p_{r e f(T R)}^{\varepsilon}\left(t_{1}+\varepsilon s_{1}\right)-p_{r e f(T R)}^{\varepsilon}\left(t_{1}+\varepsilon s_{2}\right)\right|
$$

is bounded by

$$
M^{\varepsilon}(\delta) \leq \int \sup _{\left|s_{1}-s_{2}\right| \leq \delta}\left|1-\exp \left(i \omega\left(s_{1}-s_{2}\right)\right)\right||\hat{f}(\omega)| d \omega \times \int\left|\hat{G}_{t_{1}}(h)\right| d h
$$

which goes to zero as $\delta$ goes to zero uniformly with respect to $\varepsilon$.

\subsection{Convergence of the Finite-dimensional Distributions}

The uniform boundedness (21) implies that the finite-dimensional distributions of the process $p_{r e f(T R)}^{\varepsilon}\left(t_{1}+\cdot\right)$ will be characterized by the moments

$$
\mathbb{E}\left[p_{\text {ref }(T R)}^{\varepsilon}\left(t_{1}+\varepsilon s_{1}\right)^{p_{1}} \ldots p_{\text {ref }(T R)}^{\varepsilon}\left(t_{1}+\varepsilon s_{k}\right)^{p_{k}}\right]
$$

for every real numbers $s_{1}<\ldots<s_{k}$ and every integers $p_{1}, \ldots, p_{k}$. 


\section{First Moment}

Let us first address the first moment. Using the representation (20) the expectation of $p_{r e f(T R)}^{\varepsilon}\left(t_{1}+\varepsilon t\right)$ reads:

$$
\begin{aligned}
\mathbb{E}\left[p_{\text {ref }(T R)}\left(t_{1}+\varepsilon t\right)\right]=\iint e^{i \omega t} e^{i \varepsilon h t / 2} \overline{\hat{f}}(\omega-\varepsilon h / 2) \overline{\hat{G}}_{t_{1}}(h) \\
\times \mathbb{E}\left[R^{\varepsilon}(\omega+\varepsilon h / 2,-L, 0) \overline{R^{\varepsilon}}(\omega-\varepsilon h / 2,-L, 0)\right] d h d \omega .
\end{aligned}
$$

As shown by (23), the statistical distribution of the refocused pulse will depend on the frequency autocorrelation function of the reflection coefficient. We denote:

$$
U_{1,1}^{\varepsilon}(\omega, h, z)=R^{\varepsilon}\left(\omega+\frac{\varepsilon h}{2},-L, z\right) \overline{R^{\varepsilon}}\left(\omega-\frac{\varepsilon h}{2},-L, z\right),
$$

and we extend the approach developed in [AKP91]. It is necessary to consider a family of moments so as to get a closed system of equations. We introduce for $q, p \in \mathbb{N}$

$$
U_{q, p}^{\varepsilon}(\omega, h, z)=\left(R^{\varepsilon}\left(\omega+\frac{\varepsilon h}{2},-L, z\right)\right)^{q}\left(\overline{R^{\varepsilon}}\left(\omega-\frac{\varepsilon h}{2},-L, z\right)\right)^{p} .
$$

Using the Riccati equation (16) satisfied by $R^{\varepsilon}$, we deduce

$$
\begin{aligned}
\frac{\partial U_{q, p}^{\varepsilon}}{\partial z}= & i \omega(p-q) m^{\varepsilon} U_{q, p}^{\varepsilon}+i e^{\frac{2 i \omega z}{\varepsilon}} \frac{\omega}{2}\left(-q n^{\varepsilon} e^{i h z} U_{q-1, p}^{\varepsilon}+p n^{\varepsilon} e^{-i h z} U_{q, p+1}^{\varepsilon}\right) \\
& +i e^{-\frac{2 i \omega z}{\varepsilon}} \frac{\omega}{2}\left(p n^{\varepsilon} e^{i h z} U_{q, p-1}^{\varepsilon}-q n^{\varepsilon} e^{-i h z} U_{q+1, p}^{\varepsilon}\right) \\
& -(p+q) \sigma^{\varepsilon} U_{q, p}^{\varepsilon}+e^{\frac{2 i \omega z}{\varepsilon}} \frac{\sigma^{\varepsilon}}{2}\left(q e^{i h z} U_{q-1, p}^{\varepsilon}+p e^{-i h z} U_{q, p+1}^{\varepsilon}\right) \\
& +e^{-\frac{2 i \omega z}{\varepsilon}} \frac{\sigma^{\varepsilon}}{2}\left(p e^{i h z} U_{q, p-1}^{\varepsilon}+q e^{-i h z} U_{q+1, p}^{\varepsilon}\right),
\end{aligned}
$$

starting from $U_{q, p}^{\varepsilon}(\omega, h, z=-L)=\mathbf{1}_{0}(q) \mathbf{1}_{0}(p)$, where $\mathbf{1}_{0}(q)=1$ if $q=0$ and 0 otherwise. Taking a shifted scaled Fourier transform with respect to $h$

$$
V_{q, p}^{\varepsilon}(\omega, \tau, z)=\frac{1}{2 \pi} \int e^{i h(\tau-(q+p) z)} U_{q, p}^{\varepsilon}(\omega, h, z) d h,
$$

we get

$$
\begin{aligned}
\frac{\partial V_{q, p}^{\varepsilon}}{\partial z}= & -(q+p) \frac{\partial V_{q, p}^{\varepsilon}}{\partial \tau}+i \omega(p-q) m^{\varepsilon} V_{q, p}^{\varepsilon}+i \frac{\omega}{2} e^{\frac{2 i \omega z}{\varepsilon}} n^{\varepsilon}\left(-q V_{q-1, p}^{\varepsilon}+p V_{q, p+1}^{\varepsilon}\right) \\
& +i e^{-\frac{2 i \omega z}{\varepsilon}} \frac{\omega}{2} n^{\varepsilon}\left(p V_{q, p-1}^{\varepsilon}-q V_{q+1, p}^{\varepsilon}\right)-(p+q) \sigma^{\varepsilon} V_{q, p}^{\varepsilon} \\
& +e^{\frac{2 i \omega z}{\varepsilon}} \frac{\sigma^{\varepsilon}}{2}\left(q V_{q-1, p}^{\varepsilon}+p V_{q, p+1}^{\varepsilon}\right)+e^{-\frac{2 i \omega z}{\varepsilon}} \frac{\sigma^{\varepsilon}}{2}\left(p V_{q, p-1}^{\varepsilon}+q V_{q+1, p}^{\varepsilon}\right), \quad(27)
\end{aligned}
$$


starting from $V_{q, p}^{\varepsilon}(\omega, \tau, z=-L)=\delta_{0}(\tau) \mathbf{1}_{0}(q) \mathbf{1}_{0}(p)$. A diffusion-approximation theorem [AKP91, Section 3] establishes that the processes $V_{q, p}^{\varepsilon}$ converge to diffusion processes as $\varepsilon \rightarrow 0$. In particular the expectations $\mathbb{E}\left[V_{p, p}^{\varepsilon}(\omega, \tau, z)\right]$, $p \in \mathbb{N}$, converge to $W_{p}(\omega, \tau, z)$ which obey the closed system of transport equations:

$$
\begin{aligned}
& \frac{\partial W_{p}}{\partial z}+2 p \frac{\partial W_{p}}{\partial \tau}=\left(\mathcal{L}_{\omega} W\right)_{p}-2 \bar{\sigma}(z) p W_{p}, \\
& \left(\mathcal{L}_{\omega} \phi\right)_{p}=\frac{1}{2} \alpha_{n} \omega^{2} p^{2}\left(\phi_{p+1}+\phi_{p-1}-2 \phi_{p}\right),
\end{aligned}
$$

starting from $W_{p}(\omega, \tau, z=-L)=\delta_{0}(\tau) \mathbf{1}_{0}(p)$. The parameters $\alpha_{m}$ and $\alpha_{n}$ are the integrated covariances of the processes $m$ and $n$ :

$$
\alpha_{m}=\int_{0}^{\infty} \mathbb{E}[m(0) m(z)] d z, \quad \alpha_{n}=\int_{0}^{\infty} \mathbb{E}[n(0) n(z)] d z .
$$

We then get the limit of the autocorrelation function of the reflection coefficient:

$$
\mathbb{E}\left[R^{\varepsilon}\left(\omega+\frac{\varepsilon h}{2},-L, 0\right) \overline{R^{\varepsilon}}\left(\omega-\frac{\varepsilon h}{2},-L, 0\right)\right] \stackrel{\varepsilon \rightarrow 0}{\longrightarrow} \int W_{1}(\omega, \tau, 0) e^{-i h \tau} d \tau .
$$

The quantity $W_{1}(\omega, \tau, 0)$ is obtained through the system of transport equations (28). Substituting this limit into Eq. (23) yields

$$
\begin{aligned}
\mathbb{E}\left[p_{\text {ref }(T R)}\left(t_{1}+\varepsilon t\right)\right] & \stackrel{\varepsilon \rightarrow 0}{\longrightarrow} \iiint e^{i \omega t} e^{-i h \tau} \overline{\hat{f}}(\omega) \overline{\hat{G}_{t_{1}}}(h) W_{1}(\omega, \tau, 0) d h d \tau d \omega \\
& =\iint e^{i \omega t} \overline{\hat{f}}(\omega) G_{t_{1}}(\tau) W_{1}(\omega, \tau, 0) d \tau d \omega .
\end{aligned}
$$

Remark. The existence and uniqueness of the solution $z \mapsto W .(\cdot, \cdot, z)$ to the transport equations (28) can be rigorously established in the space $\mathcal{C}\left([-L, 0], \mathcal{S}_{H}^{\prime}\right)$ where $\mathcal{S}_{H}^{\prime}$ is the space of distributions introduced in [PW94] to study an analogous system without absorption. $\mathcal{S}_{H}^{\prime}$ can be identified as the

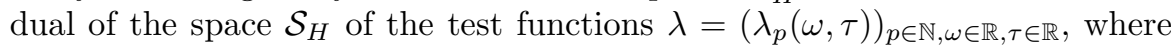
the $\lambda_{p}$ 's are infinitely differentiable in $\omega$ and $\tau$ and are rapidly decaying as functions of $\omega, \tau$, and $p$.

\section{Higher Order Moments}

Let us now consider the general moment (22). Using the representation (20) for each factor $p_{r e f(T R)}^{\varepsilon}\left(t_{1}+\varepsilon s_{j}\right)$, these moments can be written as multiple integrals over $p=\sum_{j=1}^{k} p_{j}$ frequencies:

$$
\mathbb{E}\left[\prod_{j=1}^{k} p_{r e f(T R)}^{\varepsilon}\left(t_{1}+\varepsilon s_{j}\right)^{p_{j}}\right]=\int \ldots \int \mathbb{E}\left[\prod_{\substack{1 \leq j \leq k \\ 1 \leq l \leq p_{j}}} U_{1,1}^{\varepsilon}\left(\omega_{j, l}, h_{j, l}, 0\right)\right]
$$




$$
\times \prod_{\substack{1 \leq j \leq k \\ 1 \leq l \leq p_{j}}} \overline{\hat{f}}\left(\omega_{j, l}\right) e^{i \omega_{j, l} s_{j}} e^{i \varepsilon h_{j, l} s_{j} / 2} \overline{\hat{G}_{t_{1}}}\left(h_{j, l}\right) d \omega_{j, l} d h_{j, l} .
$$

The important quantity is $\mathbb{E}\left[\prod_{j, l} U_{1,1}^{\varepsilon}\left(\omega_{j, l}, h_{j, l}, 0\right)\right]$. Our problem is now to find the limit, as $\varepsilon$ goes to 0 , of these moments for $k$ distinct frequencies. This limit will be deduced from the study of the convergence of the distribution of $\left(U_{q_{1}, p_{1}}^{\varepsilon}\left(\omega_{1}, h_{1}, z\right), \ldots, U_{q_{k}, p_{k}}^{\varepsilon}\left(\omega_{k}, h_{k}, z\right)\right)$ which results once again from the application of a diffusion-approximation theorem. Introducing $V^{\varepsilon}$ as in Eq. (26) it is found that $\left(V_{q_{1}, p_{1}}^{\varepsilon}\left(\omega_{1}, \tau_{1}, z\right), \ldots, V_{q_{k}, p_{k}}^{\varepsilon}\left(\omega_{k}, \tau_{k}, z\right)\right)$ converges as $\varepsilon \rightarrow 0$ to a diffusion process. In particular,

$$
v_{p_{1}, \ldots, p_{k}}\left(\omega_{1}, \ldots, \omega_{k}, \tau_{1}, \ldots, \tau_{k}, z\right):=\lim _{\varepsilon \rightarrow 0} \mathbb{E}\left[\prod_{j} V_{p_{j}, p_{j}}^{\varepsilon}\left(\omega_{j}, \tau_{j}, z\right)\right]
$$

is solution of

$$
\begin{aligned}
& \frac{\partial v_{p_{1}, \ldots, p_{k}}}{\partial z}+2 \sum_{j} p_{j} \frac{\partial v_{p_{1}, \ldots, p_{k}}}{\partial \tau_{j}}=\sum_{j} \mathcal{L}_{\omega_{j}} v_{p_{1}, \ldots, p_{k}}-2 \bar{\sigma}(z)\left(\sum_{j} p_{j}\right) v_{p_{1}, \ldots, p_{k}}, \\
& v_{p_{1}, \ldots, p_{k}}\left(\omega_{1}, \ldots, \omega_{k}, \tau_{1}, \ldots, \tau_{k}, z=-L\right)=\prod_{j} \delta_{0}\left(\tau_{j}\right) \mathbf{1}_{0}\left(p_{j}\right) .
\end{aligned}
$$

Using the families of processes $W$ introduced in Eq. (28) defined for every frequency $\omega$, a direct calculation shows that $\prod_{j} W_{p_{j}}\left(\omega_{j}, \tau_{j}\right)$ satisfies the above system, so that we have

$$
v_{p_{1}, \ldots, p_{k}}\left(\omega_{1}, \ldots, \omega_{k}, \tau_{1}, \ldots, \tau_{k}, z\right)=\prod_{j} W_{p_{j}}\left(\omega_{j}, \tau_{j}, z\right),
$$

and consequently,

$$
\begin{gathered}
\mathbb{E}\left[p_{r e f(T R)}^{\varepsilon}\left(t_{1}+\varepsilon s_{1}\right)^{p_{1}} \ldots p_{r e f(T R)}^{\varepsilon}\left(t_{1}+\varepsilon s_{k}\right)^{p_{k}}\right] \stackrel{\varepsilon \rightarrow 0}{\longrightarrow} \\
\int \ldots \int \prod_{\substack{1 \leq j \leq k \\
1 \leq l \leq p_{j}}} W_{1}\left(\omega_{j, l}, \tau_{j, l}, 0\right) \prod_{\substack{1 \leq j \leq k \\
1 \leq l \leq p_{j}}} \overline{\hat{f}}\left(\omega_{j, l}\right) e^{i \omega_{j, l} s_{j}} G_{t_{1}}\left(\tau_{j, l}\right) d \omega_{j, l} d \tau_{j, l} \\
=\prod_{1 \leq j \leq k}\left(\int W_{1}(\omega, \tau, 0) \hat{\hat{f}}(\omega) e^{i \omega s_{j}} G_{t_{1}}(\tau) d \omega d \tau\right)^{p_{j}} .
\end{gathered}
$$

This shows the convergence of the finite-dimensional distributions of $\left(p_{r e f(T R)}^{\varepsilon}\left(t_{1}+\right.\right.$ $\varepsilon s))_{s \in(-\infty, \infty)}$ to the ones of the deterministic function

$$
\int W_{1}(\omega, \tau, 0) \overline{\hat{f}}(\omega) e^{i \omega s} G_{t_{1}}(\tau) d \omega d \tau
$$




\subsection{Convergence of the Refocused Pulse}

We have just proved the tightness of the process $p_{\operatorname{ref}(T R)}\left(t_{1}+\varepsilon \cdot\right)$ as well as the convergence of its finite-dimensional distributions. Accordingly this proves the following theorem.

Theorem 1. The refocused signal $\left(p_{\text {ref }(T R)}\left(t_{1}+\varepsilon t\right)\right)_{t \in(-\infty, \infty)}$ converges in probability as $\varepsilon \rightarrow 0$ to the deterministic function

$$
P_{r e f(T R)}(t)=\int \Lambda(\omega, \tau) \overline{\hat{f}}(\omega) e^{i \omega t} G_{t_{1}}(\tau) d \omega d \tau,
$$

where $\Lambda(\omega, \tau)=W_{1}(\omega, \tau, 0)$ is the density given by the system (28). We can also write that

$$
P_{r e f(T R)}(t)=\left(f(-\cdot) * K_{T R}(\cdot)\right)(t) .
$$

The Fourier transform of the refocusing kernel $K_{T R}$ is given by

$$
\hat{K}_{T R}(\omega)=\int G_{t_{1}}(\tau) \Lambda(\omega, \tau) d \tau
$$

We can give a probabilistic representation of the density $\Lambda$ in terms of a jump Markov process. Let us introduce the process $\left(X_{t}\right)_{t \geq-L}$ with state space $\mathbb{N}$ and infinitesimal generator $\mathcal{L}_{\omega}$ given by (29). When the jump process reaches the state $x \in \mathbb{N}^{*}$, a random clock with exponential distribution and parameter $x^{2} \alpha_{n} \omega^{2}$ starts running. When the clock strikes, the process jumps to $x \pm 1$ with probability $1 / 2$. Finally, 0 is an absorbing state. The representation of a system of transport equations in terms of a canonical Markov process was first introduced in [AKP91], and it turns out that we can extend this idea to the system (28) by means of a Feynman-Kac formula. We get

$$
\int_{\tau_{0}}^{\tau_{1}} W_{p}(\omega, \tau, 0) d \tau=\mathbb{E}_{X_{-L}=p}\left[\mathbf{1}_{\int_{-L}^{0} 2 X_{s} d s \in\left[\tau_{0}, \tau_{1}\right]} \mathbf{1}_{X_{0}=0} e^{-\int_{-L}^{0} 2 \bar{\sigma}(-L-s) X_{s} d s}\right],
$$

where $\mathbb{E}_{X_{-L}=p}$ stands for the expectation over the distribution of the jump process starting from $X_{-L}=p$.

In the particular case of a constant mean dissipation, $\bar{\sigma}(z)=\bar{\sigma}_{0}$, the probabilistic interpretation of the densities $W_{p}$ reads:

$$
W_{p}(\omega, \tau, 0) d \tau=\mathbb{E}_{X_{-L}=p}\left[\mathbf{1}_{\int_{-L}^{0} 2 X_{s} d s \in[\tau, \tau+d \tau]} \mathbf{1}_{X_{0}=0} e^{-2 \bar{\sigma}_{0} \int_{-L}^{0} X_{s} d s}\right] .
$$

We can then note that the argument of the exponential is deterministic because the value of the integral $\int_{-L}^{0} X_{s} d s$ is constrained to be equal to $\tau / 2$. As a result,

$$
W_{p}(\omega, \tau, 0)=W_{p}^{(0)}(\omega, \tau, 0) e^{-\bar{\sigma}_{0} \tau},
$$

where $W_{p}^{(0)}$ is the solution of the transport equations (28) in absence of dissipation. We can also verify directly that (37) is indeed a solution of the transport equations $(28)$ with $\bar{\sigma}(z)=\bar{\sigma}_{0}$. 


\section{Application to the Detection of a Dissipative Layer}

In this section we prove that time reversal can be used as an efficient and statistically stable method to image a dissipative layer embedded in a randomly scattering medium. We consider the same configuration as the one studied in the previous section. We compute explicitly the refocusing kernel, we show that it contains information about the presence of an embedded dissipative layer, and we show also how this information can be extracted.

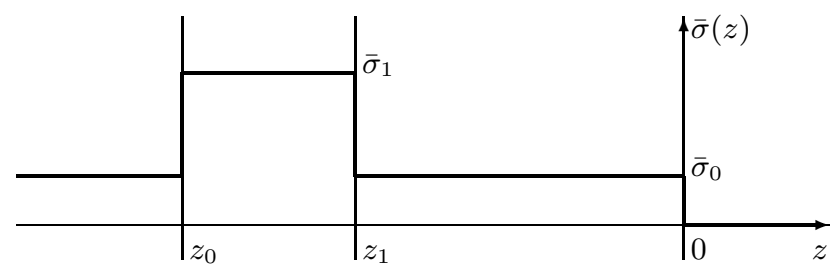

Fig. 3. Profile of the mean dissipation.

\subsection{Constant Mean Dissipation}

Here we consider the case of a half space and we assume that there is no embedded layer. The mean dissipation is constant $\bar{\sigma}(z) \equiv \bar{\sigma}_{0}$. In this configuration we can compute explicitly the solution for the system of transport equations by using (37) and the explicit solution computed in [AKP91] in absence of dissipation:

$$
W_{p}(\omega, \tau, 0)=\frac{\partial}{\partial \tau}\left[\left(\frac{\alpha_{n} \omega^{2} \tau}{4+\alpha_{n} \omega^{2} \tau}\right)^{p} \mathbf{1}_{[0, \infty)}(\tau)\right] e^{-\bar{\sigma}_{0} \tau} .
$$

We thus get a closed form expression for the density in case of constant mean dissipation:

$$
\Lambda_{0}(\omega, \tau)=\frac{4 \alpha_{n} \omega^{2}}{\left(4+\alpha_{n} \omega^{2} \tau\right)^{2}} e^{-\bar{\sigma}_{0} \tau} .
$$

Let us further assume that we record everything at the mirror, so that $G_{t_{1}} \equiv 1$. In these conditions

$$
\hat{K}_{T R}(\omega)=1-\frac{4 \bar{\sigma}_{0}}{\alpha_{n} \omega^{2}} \exp \left(\frac{4 \bar{\sigma}_{0}}{\alpha_{n} \omega^{2}}\right) E_{i}\left(\frac{4 \bar{\sigma}_{0}}{\alpha_{n} \omega^{2}}\right),
$$

where $E_{i}$ is the exponential integral function $E_{i}(x)=\int_{1}^{\infty} \exp (-x t) / t d t$. The Fourier transform of this refocusing kernel is plotted in Fig. 4. Note that $\bar{\sigma}_{0}=0$ implies that $\hat{K}_{T R}(\omega)=1$. This case is of course expected: the pulse is completely scattered back by the random half-space due to Anderson localization [Pap71], and we send back everything that is recorded, so we get 
a perfect refocusing as a result of the time-reversibility of the wave equation. In presence of dissipation, the refocusing kernel looks like a high-pass filter. This can be explained by simple arguments based on the fact that the localization length decays with increasing frequencies [Pap71]. Thus, high-frequencies (above the cut-off frequency $\omega_{c}=2 \sqrt{\bar{\sigma}_{0} / \alpha_{n}}$ ) are well recovered, because they are very quickly scattered back by the localization effect and they spend a short time in the random dissipative half-space. On the contrary, low frequencies are highly dissipated, because they penetrate deeper and spend a longer time in the medium before being scattered back.

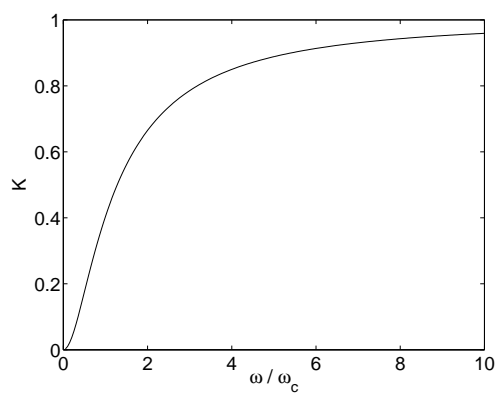

Fig. 4. Fourier transform of the refocusing kernel for a random half space with a constant mean dissipation. Here $G_{t_{1}} \equiv 1$ and $\omega_{c}=2 \sqrt{\bar{\sigma}_{0} / \alpha_{n}}$.

\subsection{Thin Dissipative Layer}

We consider here a configuration where a thin layer with mean dissipation $\bar{\sigma}_{1}$ lying in $\left[z_{0}, z_{1}\right]$ is embedded into a half plane with constant mean dissipation $\bar{\sigma}_{0}$ (see Figure 3 ). The layer is thin in the sense that $z_{0}-z_{1} \rightarrow 0$, but its dissipation coefficient is high so that $\bar{\sigma}_{1}\left(z_{0}-z_{1}\right) \rightarrow \lambda$. We will discuss the domain of validity of this limit in the next section. First, we solve the system of transport equations (28) from $-\infty$ to $z_{0}$, so that we get the stationary solution (38):

$$
W_{p}\left(\omega, \tau, z=z_{0}\right)=\frac{\partial}{\partial \tau}\left[\left(\frac{\alpha_{n} \omega^{2} \tau}{4+\alpha_{n} \omega^{2} \tau}\right)^{p} \mathbf{1}_{[0, \infty)}(\tau)\right] e^{-\bar{\sigma}_{0} \tau} .
$$

Second, we solve the system across the layer from $z_{0}$ to $z_{1}$. The layer is very thin, so that the Markov process does not jump. As a result, we get

$$
W_{p}\left(\omega, \tau, z=z_{1}\right)=\frac{\partial}{\partial \tau}\left[\left(\frac{\alpha_{n} \omega^{2} \tau}{4+\alpha_{n} \omega^{2} \tau}\right)^{p} \mathbf{1}_{[0, \infty)}(\tau)\right] e^{-\bar{\sigma}_{0} \tau-2 \lambda p} .
$$

Third, we solve the system from $z_{1}$ to 0 with the initial condition given by $W_{p}\left(\omega, \tau, z=z_{1}\right)$. Using the probabilistic interpretation of the solution of the 
system transport equations, we obtain that the density $\Lambda(\omega, \tau)=W_{1}(\omega, \tau, z=$ $0)$ is

$$
\Lambda(\omega, \tau)=\mathbb{E}_{X_{z_{1}}=1}\left[W_{X_{0}}\left(\omega, \tau-2 \int_{z_{1}}^{0} X_{s} d s, z_{1}\right)\right]
$$

This expression would be equal to the stationary solution (38) if the multiplicative factor $\exp \left(-2 \lambda X_{0}\right)$ was absent. We then expand this factor as $1-\left(1-\exp \left(-2 \lambda X_{0}\right)\right)$ so that we obtain

$$
\Lambda(\omega, \tau)=\Lambda_{0}(\omega, \tau)-\mathbb{E}_{X_{z_{1}}=1}\left[\tilde{W}_{X_{0}}^{(0)}\left(\omega, \tau-2 \int_{z_{1}}^{0} X_{s} d s\right)\left(1-e^{-2 \lambda X_{0}}\right)\right] e^{-\bar{\sigma}_{0} \tau}
$$

where the expectation $\mathbb{E}_{1}$ is taken with respect to the distribution of the jump Markov process $X_{z}$ starting from $X_{z_{1}}=1$, and

$$
\tilde{W}_{p}^{(0)}(\omega, \tau)= \begin{cases}\delta_{0}(\tau) & \text { if } p=0 \\ 4 p \alpha_{n} \omega^{2} \frac{\left(\alpha_{n} \omega^{2} \tau\right)^{p-1}}{\left(4+\alpha_{n} \omega^{2} \tau\right)^{p+1}} \mathbf{1}_{[0, \infty)}(\tau) & \text { otherwise }\end{cases}
$$

Let us have a look at the expectation in the right-hand side of Eq. (40). If $X_{0}=$ 0 , then the second term inside the expectation is zero. If $X_{0} \geq 1$, then $X_{z} \geq 1$ for all $z \in\left[z_{1}, 0\right]$ because 0 is an absorbing state. This means that only the paths which never reach zero can contribute to the value of the expectation. These paths satisfy $\int_{z_{1}}^{0} X_{s} d s \geq\left|z_{1}\right|$. Furthermore, $\tilde{W}_{p}^{(0)}(\omega, \tau)=0$ for $\tau<$ 0 . This shows that the expectation is zero for any $\tau<2\left|z_{1}\right|$. Accordingly, the density $\Lambda$ is indistinguishable from the density $\Lambda_{0}$ corresponding to a constant mean dissipation for any $\tau \leq 2\left|z_{1}\right|$. When $\tau$ crosses this critical value corresponding to a round trip form the surface to the layer, a density jump occurs. Indeed a set of paths suddenly contributes to the expectation in the right-hand side of Eq. (40). This is the set of paths where no jump occurs (i.e. $X_{z}=1$ for all $z \in\left[z_{1}, 0\right]$ ). The density then jumps from

$$
\Lambda\left(\omega, \tau=2\left|z_{1}\right|^{-}\right)=\Lambda_{0}\left(\omega, 2\left|z_{1}\right|\right)
$$

to

$$
\begin{aligned}
\Lambda\left(\omega, \tau=2\left|z_{1}\right|^{+}\right)= & \Lambda_{0}\left(\omega, 2\left|z_{1}\right|\right) \\
& -\tilde{W}_{1}^{(0)}(\omega, 0)\left(1-e^{-2 \lambda}\right) \mathbb{P}_{1}(\text { no jump before } 0) e^{-\bar{\sigma}_{0} \tau} \\
= & \Lambda_{0}\left(\omega, 2\left|z_{1}\right|\right)[1-\Delta \Lambda]
\end{aligned}
$$

where the relative amplitude of the jump is

$$
\Delta \Lambda=\left(1-e^{-2 \lambda}\right)\left(1+\frac{\alpha_{n} \omega^{2}}{2}\left|z_{1}\right|\right)^{2} e^{-\alpha_{n} \omega^{2}\left|z_{1}\right|}
$$

Summary: in order to detect the depth and the dissipation strength of the layer from a measured density $\Lambda$, one can plot the ratio of the measured density 
$\Lambda$ over $\Lambda_{0}$. The ratio is 1 up to $\tau=2\left|z_{1}\right|$. A density jump occurs at $2\left|z_{1}\right|$. The amplitude of the jump is (42) and allows us to recover the dissipation strength $\lambda$.

We have carried out MC simulations of the jump Markov process $X$ to compute $\Lambda$ from the expression (40). The results for several sets of parameters are plotted in Fig. 5 where it can be checked that the jump of the density can be clearly detected. The computation of each density profile requires $N=10^{6}$ simulations.

Remark. We can estimate the confidence interval for the MC simulations. Indeed the quantity to be estimated is roughly speaking the probability of a rare event $p \simeq 10^{-2}-10^{-1}$. In such a case the relative error of a standard MC method is about $1 / \sqrt{N p}$ which is of the order of a few percents.

(a)

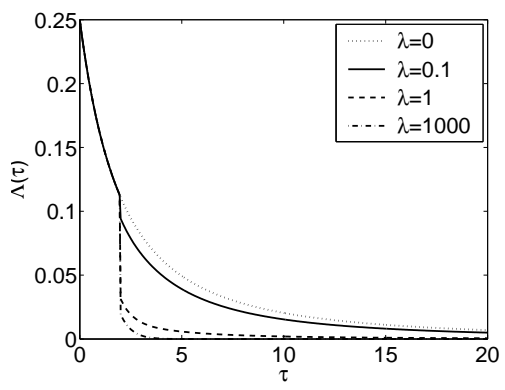

(b)

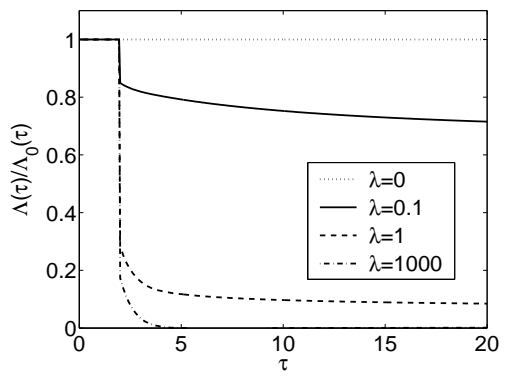

Fig. 5. Picture a: Density $\tau \mapsto \Lambda(\omega, \tau)$. Picture b: Ratio of the densities $\tau \mapsto$ $\Lambda(\omega, \tau) / \Lambda_{0}(\omega, \tau)$. Here we assume $\alpha_{n} \omega^{2}=1, z_{0}=1$.

\subsection{Thick Dissipative Layer}

We revisit the previous configuration without assuming that the layer is thin. Accordingly we consider a configuration where a layer with mean dissipation $\bar{\sigma}_{1}$ lying in $\left[z_{0}, z_{1}\right]$ is embedded into a half plane with mean dissipation $\bar{\sigma}_{0}$. We proceed as above and establish that

$$
\begin{aligned}
\Lambda(\omega, \tau)=\mathbb{E}_{X_{z_{0}}=1} & \left\{W_{X_{0}}\left(\omega, \tau-2 \int_{z_{0}}^{0} X_{s} d s, z_{0}\right)\right. \\
& \left.\times \exp \left(2\left(\bar{\sigma}_{0}-\bar{\sigma}_{1}\right) \int_{z_{0}-z_{1}}^{0} X_{s} d s\right)\right\} e^{-\bar{\sigma}_{0} \tau} \\
=\Lambda_{0}(\omega, \tau) & -\mathbb{E}_{X_{z_{0}}=1}\left\{\tilde{W}_{X_{0}}^{(0)}\left(\omega, \tau-2 \int_{z_{0}}^{0} X_{s} d s\right)\right. \\
& \left.\times\left[1-\exp \left(2\left(\bar{\sigma}_{0}-\bar{\sigma}_{1}\right) \int_{z_{0}-z_{1}}^{0} X_{s} d s\right)\right]\right\} e^{-\bar{\sigma}_{0} \tau} .
\end{aligned}
$$


If $X_{z_{0}-z_{1}}=0$, then $X_{z}=0$ for all $z \in\left[z_{0}-z_{1}, 0\right]$ and the second term inside the expectation is zero. If $X_{z_{0}-z_{1}} \geq 1$, then $X_{z} \geq 1$ for all $z \in\left[z_{0}, z_{0}-z_{1}\right]$, so that $2 \int_{z_{0}}^{0} X_{s} d s \geq 2\left|z_{1}\right|$. The fact that $\tilde{W}_{p}^{(0)}(\omega, \tau)$ is zero for $\tau<0$ then shows that the first term inside the expectation is zero if $\tau \leq 2\left|z_{1}\right|$. Accordingly $\Lambda(\omega, \tau)=\Lambda_{0}(\omega, \tau)$ for $\tau \leq 2\left|z_{1}\right|$.

There is a jump of the derivative of the density at $\tau=2\left|z_{1}\right|$. Indeed, for $\tau$ just above $2\left|z_{1}\right|$ a path contributes to the value of the expectation that appears in the right-hand side of Eq. (43), namely the path where $X_{z}=1$ for all $z \in\left[z_{0}, z_{0}-z_{1}\right]$ and a jump from state 1 to state 0 occurs at $z_{0}+\tau / 2$. Accordingly, the derivative goes from

$$
\begin{aligned}
\Lambda^{\prime}\left(\omega, \tau=2\left|z_{1}\right|^{-}\right) & =\Lambda_{0}^{\prime}\left(\omega, 2\left|z_{1}\right|\right) \\
& =-\frac{\alpha_{n} \omega^{2}}{\left(2+\alpha_{n} \omega^{2}\left|z_{1}\right|\right)^{3}}\left(\alpha_{n} \omega^{2}+\bar{\sigma}_{0}\left(2+\alpha_{n} \omega^{2}\left|z_{1}\right|\right)\right) e^{-2 \bar{\sigma}_{0}\left|z_{1}\right|}
\end{aligned}
$$

to

$$
\begin{aligned}
\Lambda^{\prime}\left(\omega, \tau=2\left|z_{1}\right|^{+}\right) & =\Lambda_{0}^{\prime}\left(\omega, 2\left|z_{1}\right|\right)-\frac{1}{4}\left(\bar{\sigma}_{1}-\bar{\sigma}_{0}\right) \alpha_{n} \omega^{2} e^{-\alpha_{n} \omega^{2}\left|z_{1}\right|} e^{-2 \bar{\sigma}_{0}\left|z_{1}\right|} \\
& =\Lambda_{0}^{\prime}\left(\omega, 2\left|z_{1}\right|\right)\left[1+\Delta \Lambda^{\prime}\right]
\end{aligned}
$$

where the relative amplitude of the jump is

$$
\Delta \Lambda^{\prime}=\frac{\bar{\sigma}_{1}-\bar{\sigma}_{0}}{4} \frac{\left(2+\alpha_{n} \omega^{2}\left|z_{1}\right|\right)^{3}}{\alpha_{n} \omega^{2}+\bar{\sigma}_{0}\left(2+\alpha_{n} \omega^{2}\left|z_{1}\right|\right)} e^{-\alpha_{n} \omega^{2}\left|z_{1}\right|} .
$$

There is a second jump in the derivative of $\Lambda$ at $\tau=2\left|z_{0}\right|$. Indeed the mechanism described just above fails precisely when $\tau$ become larger than $\tau=2\left|z_{0}\right|$, because the jump of the Markov process at $z_{0}+\tau / 2>0$ has no influence.

Summary: in order to detect the depth, the thickness, and the dissipation coefficient of the layer from a measured density $\Lambda$, one can plot the ratio of the measured density $\Lambda$ over $\Lambda_{0}$. The ratio is 1 up to $\tau=2\left|z_{1}\right|$. The position of the first jump in the derivative of the density is $2\left|z_{1}\right|$, the position of the second jump is $2\left|z_{0}\right|$. The amplitude of the first jump is (44) and allows us to recover $\bar{\sigma}_{1}$. Note that we need to know the background dissipation $\bar{\sigma}_{0}$ to get $\bar{\sigma}_{1}$, as we detect the difference $\bar{\sigma}_{1}-\bar{\sigma}_{0}$.

We have carried out Monte Carlo simulations of the jump Markov process $X$ to compute $\Lambda$ from the expression (43). The results for several sets of parameters are plotted in Fig. 6 . Each density profile requires $10^{7}$ simulations. It can be checked that the first jump of the derivative density can be clearly detected. It may be more difficult to detect the second jump if the dissipative layer is very thick. Note that the case where the thickness of the layer is very small $z_{1}-z_{0}=0.1$ is very similar to the approximation of a thin layer with $\lambda=0.1$ presented in Fig. 5 .

The thin layer approximation developed in Section 6.2 can now be discussed more quantitatively. The interpretation in terms of the jump Markov 
(a)

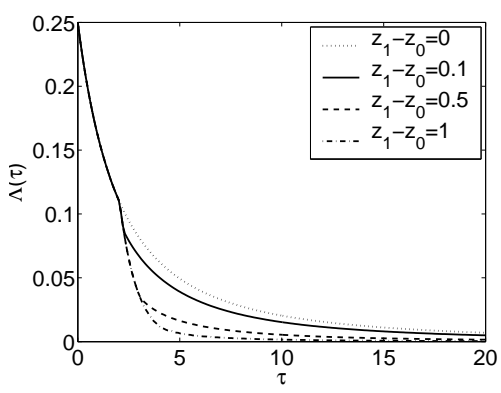

(b)

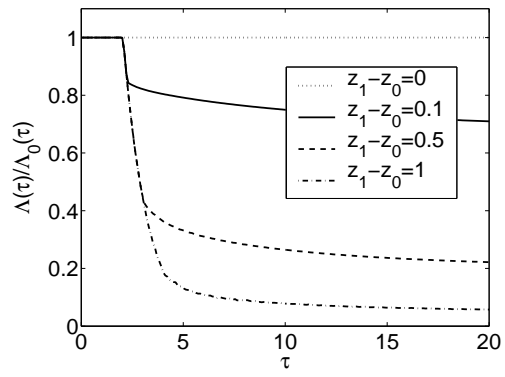

Fig. 6. Picture a: Density $\tau \mapsto \Lambda(\omega, \tau)$. Picture b: Ratio of the densities $\tau \mapsto$ $\Lambda(\omega, \tau) / \Lambda_{0}(\omega, \tau)$. Here we assume $\alpha_{n} \omega^{2}=1, z_{1}=1, \bar{\sigma}_{0}=0, \bar{\sigma}_{1}=1$, and the thickness of the layer $z_{1}-z_{0}$ goes from 0 (absence of dissipative layer) to 1 .

is helpful for this discussion. Considering expression (43), it can be seen that the approximation holds true if the event "the process jumps between $z_{0}-z_{1}$ and 0" is negligible. The brackets [.] in the right-hand side of Eq. (43) then simplifies into $\left[1-\exp \left(2\left(\bar{\sigma}_{0}-\bar{\sigma}_{1}\right)\left(z_{1}-z_{0}\right) X_{0}\right)\right]$ and we recover precisely Eq. (40). This event is negligible if $\alpha_{n} \omega^{2}\left|z_{1}-z_{0}\right| \ll 1$ and this condition turns out to be the criterion for the validity of the thin layer approximation.

\section{Acknowledgments}

J.-P. Fouque was supported by the ONR grant N00014-02-1-0089. J. Garnier acknowledges support from the French program ACI-NIM-2003-94. A. Nachbin was supported by CNPq/Brazil under grant 300368/96-8. K. Sølna was supported by the Darpa grant N00014-02-1-0603 and by the NSF grant 0307011.

\section{References}

[AKP91] Asch, M., Kohler, W., Papanicolaou, G., Postel, M., White, B.: Frequency content of randomly scattered signals. SIAM Rev., 33, 519-625 (1991).

[BPR02] Bal, G., Papanicolaou, G., Ryzhik, L.: Self-averaging in time reversal for the parabolic wave equation. Stochastics and Dynamics, 2, 507-531 (2002).

[BPZ02] Blomgren, P., Papanicolaou, G., Zhao, H.: Super-resolution in time-reversal acoustics. J. Acoust. Soc. Am., 111, 230-248 (2002).

[CF97] Clouet, J.F., Fouque, J.P.: A time-reversal method for an acoustical pulse propagating in randomly layered media. Wave Motion, 25, 361-368 (1997).

[DTR03] Derode, A., Tourin, A., de Rosny, J., Tanter, M., Yon, S., Fink, M.: Taking advantage of multiple scattering to communicate with time reversal antennas. Phys. Rev. Lett., 90, 014301 (2003). 
[Fin99] Fink, M.: Time reversed acoustics. Scientific American, 281:5, 91-97 (1999).

[FMT03] Fink, M., Montaldo, G., Tanter, M.: Time reversal acoustics in biomedical engineering. Annual Review of Biomedical Engineering, 5, 465-497 (2003).

[FS03] Fouque, J.P., Sølna, K.: Time-reversal aperture enhancement. SIAM Multiscale Modeling and Simulation, 1, 239-259 (2003).

[Kus84] Kushner, H.J.: Approximation and Weak Convergence Methods for Random Processes. MIT Press, Cambridge (1984).

[Pap71] Papanicolaou, G.: Wave propagation in a one-dimensional random medium. SIAM J. Appl. Math., 21, 13-18 (1971).

[Pap78] Papanicolaou, G.: Asymptotic analysis of stochastic equations. In: Rosenblatt, A. (ed), MAA Stud. in Math., 18, 111-179 (1978).

[PRS03] Papanicolaou, G., Ryzhik, L., Sølna, K.: Statistical stability in time reversal. SIAM J. Appl. Math., 64, 1133-1155 (2004).

[PW94] Papanicolaou, G., Weinryb, S.: A functional limit theorem for waves reflected by a random medium. Appl. Math. Optim., 30, 307-334 (1994).

[PKC02] Prada, C., Kerbrat, E., Cassereau, D., Fink, M.: Time reversal techniques in ultrasonic nondestructive testing of scattering media. Inverse Problems, 18, 1761-1773 (2002).

[Sol03] Sølna, K.: Focusing of time-reversed reflections. Waves in Random Media, 12, 365-385 (2003). 